\title{
Pre-treatment with Concanavalin-A increases resistance of mice to peritoneal infection by Serratia marcescens
}

\author{
S. M. A. A. ARRAES, L. C. J. GAZIRI†, M. C. VIDOTTO*, H. O. SARIDAKIS* and I. FELIPE* \\ Laboratory of Clinical Immunology, Universidade Estadual de Maringá, 87020-900 Maringá, \\ * Departments of General Pathology and †Physiological Sciences, Universidade Estadual de Londrina, 86051 - \\ 970 Londrina, Brazil
}

Mice pre-treated with Concanavalin-A largely survived an intra-peritoneal inoculum of $2 \times 10^{7}$ Serratia marcescens, whereas all control mice died within $15 \mathrm{~h}$ of inoculation. A subpopulation of peritoneal macrophages from Con-A pre-treated mice was able to phagocytose the bacteria in vitro (6.7 SEM 1.2\% phagocytosing cells) and in vivo (16.9 SEM 2.1\%), whereas control phagocytes did not phagocytose $S$. marcescens. The survival of Con-A pre-treated mice allowed their immunisation with living bacteria, and the antiserum thus produced increased the phagocytosis of $S$. marcescens in vitro. Control mice largely survived an inoculum of $S$. marcescens suspended in $50 \%$ immune serum, although the bacteria were resistant to the bactericidal activity of that serum. These results suggest that, in contrast to the delayed humoral protection afforded by immunisation, phagocytosis by phagocytes activated by Con-A conferred early protection to mice against experimental infection by $S$. marcescens.

\section{Introduction}

Resistance to the bactericidal activity of normal serum and to phagocytosis probably constitute important virulence factors of the gram-negative bacterium Serratia marcescens. The complement-mediated bactericidal activity of normal serum depends on the covalent fixation of $\mathrm{C} 3 \mathrm{~b}$ to the target cell surface. A strain of $S$. marcescens resistant to serum bound less $\mathrm{C} 3 \mathrm{~b}$ than did a sensitive strain [1]; this difference in serum sensitivity was attributed to the presence of long $O$ antigen (LPS) chains on the surface of the resistant strain, that prevented complement binding. A similar correlation between the presence of $\mathrm{O}$ antigen and resistance to serum has been reported for several gramnegative bacteria [2-4]. On the other hand, the resistance to phagocytosis has often been correlated with the presence of $\mathrm{K}$ (capsular) antigens [5-7], whose role in $S$. marcescens resistance to phagocytosis is unclear. Nevertheless, an analysis of the immunogenic properties of $S$. marcescens outer membrane has demonstrated that most antigenic sites of the bacterial

Received 8 Aug. 1995; revised version received 30 June 1996; accepted 20 Aug. 1996.

Corresponding author: Professor I. Felipe. surface are unable to react with antibodies and are possibly masked by the $\mathrm{O}$ antigen [8].

Mice challenged with a sublethal intraperitoneal inoculum of Escherichia coli later showed resistance to a lethal dose of the bacteria [9]; the observed increase in bacterial destruction during the early phase of infection was ascribed to non-specific activation of phagocytes. Similar results were observed following intravenous vaccination of mice with formalin-killed $S$. marcescens [10], also leading to the conclusion that enhancement of host defence in the early phase of infection depended on non-specific activation of phagocytic functions. However, those studies employing vaccination are inadequate in providing a clear distinction between early humoral immunity and nonspecific activation of phagocytosis. A later study [11] showed that effective protection of mice vaccinated with formalin-killed $S$. marcescens was achieved only by the additive effect of humoral immunity and T-cell mediated immunity.

Previous studies demonstrated that the pre-treatment of mice with either concanavalin-A (Con-A) or jacalin increased the rate of clearance of Candida albicans from the peritoneal cavity, increased the phagocytosis of the yeast by peritoneal macrophages, and decreased 
its dissemination to the viscera [12]; these effects were ascribed to non-specific activation of cell-mediated immunity (CMI) by Con-A and by jacalin. Because activation of CMI by Con-A avoids the exposure to bacterial antigens employed in vaccination studies, this investigation tested the hypothesis that Con-A could induce phagocytosis of $S$. marcescens in the early stage of infection and thus confer protection to mice challenged with these bacteria.

\section{Materials and methods}

\section{Bacteria and growth conditions}

Serratia marcescens strain O6, 14:H4 was isolated from blood of a patient with nosocomial infection at the University Hospital, Faculdade de Medicina de Ribeirão Preto, SP, Brazil, and was serotyped by Instituto Adolfo Lutz, São Paulo, Brazil. The bacteria were grown overnight in Tryptic Soy Broth (TSB; Difco) at $37^{\circ} \mathrm{C}$. When the culture reached the required optical density (OD), the bacteria were harvested and transferred to the medium employed in the phagocytic assays. Three dilutions of each culture were plated on agar to determine the number of cfu, to confirm that it corresponded to the bacterial concentration expected from the OD measurement.

\section{Peritoneal phagocytic cells}

Peritoneal phagocytes were collected from Swiss mice (weighing 25-30 g) that had received $500 \mu \mathrm{g}$ of Con-A (Sigma) in $0.5 \mathrm{ml}$ of phosphate buffered saline (PBS), or this vehicle only, via the intraperitoneal route 4 days earlier. The animals were killed with ether and the phagocytes were collected by washing the peritoneal cavity with $3 \mathrm{ml}$ of RPMI medium (Roswell Park Memorial Institute medium for cell culture; Sigma) with albumin $1 \%$. The phagocytes were allowed to adhere to coverslips for $1 \mathrm{~h}$ at $37^{\circ} \mathrm{C}$, and the monolayers were then washed in RPMI medium and used in the phagocytic assays.

\section{Phagocytosis assay}

Monolayers of phagocytic cells were incubated with $2 \times 10^{7}$ cfu of $S$. marcescens for $1 \mathrm{~h}$ at $37^{\circ} \mathrm{C}$ in $1 \mathrm{ml}$ of RPMI medium buffered to $\mathrm{pH} 7.0$ with $12 \mathrm{mM}$ Hepes. The phagocyte preparations were then washed with RPMI medium, incubated for $15 \mathrm{~min}$ with gentamicin $100 \mathrm{mg} / \mathrm{L}$ to remove non-ingested bacteria $[13,14]$, washed with PBS, fixed in absolute methanol for $15 \mathrm{~min}$, and stained with haematoxylin eosin. For each preparation 200 cells were counted to evaluate both the percentage of cell types in the peritoneal exudate and the phagocytic index (PI); this index was calculated as the percentage of phagocytosing cells multiplied by the average number of internalised particles. As a control of the phagocytic activity of each sample of macrophages, in each experiment duplicate monolayers of phagocytic cells were incubated with either $10^{6} \mathrm{cfu}$ of C. albicans or $0.5 \%$ sheep erythrocytes that had been opsonised with rabbit anti-sheep IgG $[15,16]$. Results were reported as mean PI and SEM; Student's $t$ test was employed for statistical analysis.

\section{Assay of in-vivo phagocytosis and determination} of the survival rate

Swiss mice, weighing $25-30 \mathrm{~g}$ and 6-8-weeks old, were used in these experiments. One group of mice received $500 \mu \mathrm{g}$ of Con-A in $0.5 \mathrm{ml}$ of $\mathrm{PBS}$ and the other $0.5 \mathrm{ml}$ of PBS, administered in a single intraperitoneal dose $96 \mathrm{~h}$ before inoculation with $2 \times$ $10^{7} \mathrm{cfu}$ of an exponential culture of $S$. marcescens; $1 \mathrm{~h}$ after the intraperitoneal inoculation of $S$. marcescens, the mice were killed and the peritoneal exudate cells were collected and processed for analysis of phagocytosis in vivo. The spleen, liver and kidneys were removed and plated on TSB-agar to evaluate the dissemination of the bacteria.

A group of 17 mice pre-treated with Con-A received an inoculum as above and was used for the determination of the survival rate; the control group of eight mice received an intraperitoneal inoculum of $S$. marcescens $96 \mathrm{~h}$ after an intraperitoneal injection of PBS $0.5 \mathrm{ml}$.

\section{Immunisation procedure}

The 13 mice pretreated with Con-A that survived the inoculation of $S$. marcescens were further immunised by two intraperitoneal injections of $2 \times 10^{7} \mathrm{cfu}$ of viable $S$. marcescens, with an interval of 15 days between each inoculation. The mice were bled 7 days after the last boost and their sera were pooled; the antibody titre of the sera was determined by bacterial agglutination. $\mathrm{O}$-antigen and $\mathrm{H}$-antigen preparations of the $S$. marcescens strain [17] reacted with the antisera at a dilution of 1600 . The sera of control mice did not cause agglutination of $S$. marcescens at 1 in 1 dilution.

\section{Serum resistance assay}

Blood collected from normal mice was allowed to clot at $4^{\circ} \mathrm{C}$ for $2 \mathrm{~h}$, centrifuged at $4^{\circ} \mathrm{C}$, and the separated serum was used immediately. The bacterial resistance to the lethal activity of complement was determined by a rapid turbidimetric assay in microtitration plates [18], with modification. Overnight bacterial cultures grown in TSB were diluted 1 in 100 in freshly prepared TSB medium, incubated at $37^{\circ} \mathrm{C}$ for $90 \mathrm{~min}$ with shaking, centrifuged $(2500 \mathrm{~g}, 15 \mathrm{~min})$, resuspended in cold PBS, pH $7.4\left(10^{7} \mathrm{cfu} / \mathrm{ml}\right)$, and kept in an ice bath for prompt use. The bacterial suspension was then pipetted into the wells of a microtitration plate, and either normal or immune mouse serum was added to a final concentration of $50 \%$. The plates were shaken briefly and incubated at $37^{\circ} \mathrm{C}$; absorbances of triplicates of the samples were read at $0,60,120$ and $180 \mathrm{~min}$ in a 
spectrophotometer (Titertek Multiskan, model 340) at $620 \mathrm{~nm}$. The serum-sensitive strain E. coli K12-711 was used as control.

\section{Results}

Effect of pre-treatment with Con-A on phagocytosis of $S$. marcescens

$S$. marcescens bacteria were not phagocytosed by resident peritoneal macrophages either in the absence or in the presence of mouse serum 10\% (Table 1). Phagocytosis of $C$. albicans and of opsonised sheep erythrocytes was used as a control of the phagocytic activity of each sample of phagocytes, and it was observed that serum significantly increased the phagocytosis of these cells. On the other hand, macrophages from mice pre-treated with Con-A were able to phagocytose $S$. marcescens both in vitro and in vivo (Table 1). A subpopulation of macrophages capable of ingesting $S$. marcescens was increased in number by the pre-treatment of mice with Con-A (Table 2).

\section{Effect of Con-A on mouse survival and immunisation}

The $S$. marcescens strain was virulent for untreated mice; all eight died within $15 \mathrm{~h}$ of bacterial inoculation. However, 13 of the 17 mice pre-treated with ConA survived the inoculation of $S$. marcescens and were subsequently immunised with two additional inocula of $2 \times 10^{7}$ viable bacteria. The survival rate of untreated mice was increased from 0 to $75 \%(6 / 8)$ by preincubating the bacterial inoculum for $2 \mathrm{~h}$ at $37^{\circ} \mathrm{C}$ with immune serum diluted 1 in 2 .

The peritoneal fluid was intensely haemorrhagic in a second group of eight control mice killed $1 \mathrm{~h}$ after bacterial inoculation, and there was a reduction of $c$. 25 times in the number of cells collected from their peritoneal cavity compared with the mean of $2.6 \times 10^{6} \mathrm{cell} \mathrm{s} / \mathrm{ml}$ observed in the non-inoculated control mice and in previous studies [12]. Although the strain of $S$. marcescens used in this study was consistently non-haemolytic both on solid and in liquid assay media, the peritoneal fluid collected from
Table 2. Effect of pre-treatment with Con-A on the number of cells phagocytosing $S$. marcescens

\begin{tabular}{lcc}
\hline & \multicolumn{2}{c}{ Mean (SEM) phagocytosing cells (\%) } \\
\cline { 2 - 3 } Conditions & in vitro & in vivo \\
\hline Control & 0 & 0 \\
Con-A & $6.7(1.2)$ & $16.9(2.1)$ \\
\hline
\end{tabular}

The phagocytic assays in vitro were carried out with phagocytes collected from mouse peritoneal cavity and incubated for $1 \mathrm{~h}$ with the bacteria. For the assays in vivo, the phagocytes were collected $1 \mathrm{~h}$ after inoculation of $S$. marcescens into the peritoneal cavity. Con-A: mice were treated with $500 \mu \mathrm{g}$ of Con-A 4 days before the assays. The values are mean (SEM) of at least four assays.

these group of mice presented a large number of ruptured macrophages, cell nuclei and lysed erythrocytes. On the other hand, the mean number of phagocytes harvested from Con-A pre-treated mice was increased to a mean of $1.2 \times 10^{7}$ cells $/ \mathrm{ml} 1 \mathrm{~h}$ after inoculation of the bacteria, as observed in the studies of phagocytosis in vivo.

\section{Titres of the immune antiserum}

The pooled serum from the immunised mice agglutinated $S$. marcescens to a titre of 800 . The antiserum yielded a titre of 1600 against an $\mathrm{O}$-antigen and $\mathrm{H}$ antigen preparation of the $S$. marcescens strain. Neat serum from control mice did not agglutinate the $S$. marcescens.

\section{Effect of immune serum on the phagocytosis of S. marcescens}

Resident peritoneal macrophages phagocytosed $S$. marcescens in a medium containing the immune serum diluted in 1 in 1000 , whereas in a medium containing control mouse serum they failed to phagocytose the bacteria (Fig. 1). Addition of control mouse serum to the medium containing the antiserum caused no further increase in phagocytosis ( $p>0.05$, Fig. 1).

\section{Sensitivity of S. marcescens to the bactericidal effect of serum}

S. marcescens was resistant to the lytic action of $50 \%$ normal or immune mouse serum, as shown in Fig. 2.

Table 1. Phagocytosis of $S$. marcescens by resident and by Con-A-activated phagocytes

\begin{tabular}{|c|c|c|c|c|}
\hline \multirow[b]{3}{*}{ Phagocytosis target } & \multicolumn{4}{|c|}{ Mean (SEM) phagocytic index of macrophages } \\
\hline & \multirow{2}{*}{$\begin{array}{l}\text { Resident } \\
\text { in vitro }\end{array}$} & \multirow{2}{*}{$\begin{array}{l}\text { Resident* } \\
\text { in vitro }\end{array}$} & \multicolumn{2}{|c|}{ Con-A treated } \\
\hline & & & in vitro & in vivo \\
\hline S. marcescens & 0 & 0 & $12.7(4.1)$ & $36.0(14.7)$ \\
\hline C. albicans & $19.9(6.2)$ & $144.7(18.5)$ & $97.7(16.5)$ & 41.2 \\
\hline ElgG & $11.1(3.4)$ & $125.2(29.4)$ & ND & ND \\
\hline
\end{tabular}




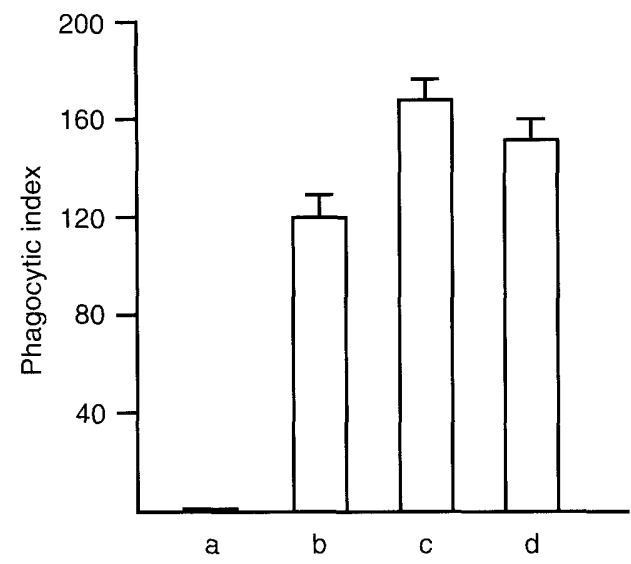

Fig. 1. Effect of antiserum against $S$. marcescens on its phagocytosis by non-activated peritoneal phagocytes. Phagocytic assays were carried out in: a, RPMI medium containing $2 \times 10^{7} \mathrm{cfu}$ of $S$. marcescens (Phagocytic index $=0$ ); or in the same medium supplemented with b, antiserum against $S$. marcescens (1:1000), c, antiserum plus fresh normal serum $10 \%$, d, antiserum plus heat inactivated normal serum $10 \%$. The differences between the means of $b, c$, or $d$ were not statistically significant $(p>0.05)$. Bars represent SEM of four experiments.

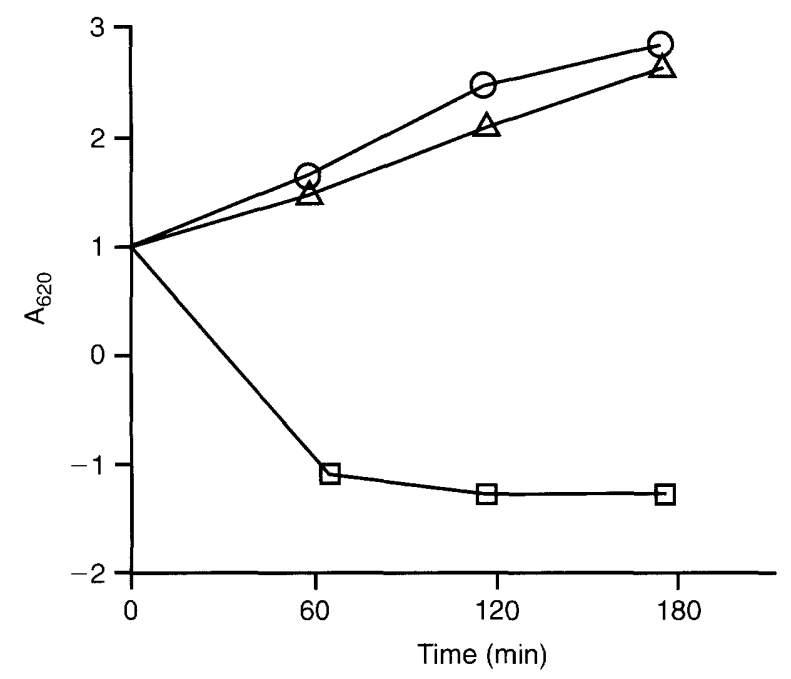

Fig. 2. Resistance of $S$. marcescens to the bactericidal activity of normal $(\triangle)$ or immune $(O)$ serum; serumsensitive E. coli $\mathrm{K} 12$ was used as control ( $\square$ ). Each symbol represents the mean of four experiments.

Escherichia coli $\mathrm{K} 12$, which is sensitive to lysis by complement, was used as a control.

\section{Discussion}

Peritoneal macrophages from mice pre-treated with Con-A were able to phagocytose $S$. marcescens both in vivo and in vitro, whereas resident phagocytes from control mice were not. However, these resident macrophages were capable of phagocytosing either opsonised sheep erythrocytes or C. albicans, which shows that they were capable both of opsonophagocytosis and lectinophagocytosis. Moreover, addition of fresh mouse serum to the phagocytic assay did not cause complement-mediated phagocytosis of the bacteria by resident phagocytes, in agreement with a previous observation [1] that a strain of $S$. marcescens resistant to serum (as was the one used in this study) bound less $\mathrm{C} 3 \mathrm{~b}$ than a sensitive strain. Addition of cellfree exudate collected from the peritoneal cavity of mice pre-treated with Con-A to the in-vitro phagocytic assay was also ineffective in causing phagocytosis by control phagocytes (data not shown). As the macrophages used in the in-vivo assay were collected shortly (1 h) after inoculation of the bacteria, these results show that the early phagocytosis of $S$. marcescens can be attributed to an activation of phagocytes by Con-A and not to humoral immunity. Furthermore, macrophages collected from mice pre-treated with Con-A, but not inoculated with $S$. marcescens, phagocytosed the bacteria in vitro, corroborating the conclusion that a subpopulation of macrophages activated by Con-A was responsible for the phagocytosis of $S$. marcescens.

Mice pre-treated with Con-A survived an otherwise lethal inoculum of $S$. marcescens, whereas control mice died within $15 \mathrm{~h}$ of inoculation. This short survival period of the control mice suggests that the protection afforded by Con-A can be attributed to the early activation of phagocytosis discussed above. ConA induces secretion of interferon gamma (IFN- $\gamma$ ) and other cytokines, and consequently causes activation of the bactericidal potential of macrophages $[19,20]$. Therefore, it seems likely that a particular subset of phagocytes (Table 2) was activated by Con-A-induced release of IFN- $\gamma$, and that this subset of phagocytes was responsible for the protection of mice against a lethal dose of $S$. marcescens.

The antiserum obtained from Con-A pre-treated mice that survived bacterial inoculation was effective in the opsonisation of $S$. marcescens, as shown by its induction of phagocytosis in vitro by resident macrophages. As immune complexes of IgG and IgM can bind to rabbit polymorphonuclear leucocytes [21], but only the $\operatorname{IgG}$ complexes are readily phagocytosed, these results suggest that $\operatorname{IgG}$ present in the immune serum mediated the phagocytosis of $S$. marcescens by non-activated phagocytes. Furthermore, pre-incubation of $S$. marcescens with the antiserum protected control mice against a lethal dose of the bacteria; because the bacteria were resistant to the lytic activity of $50 \%$ serum, either normal or immune, this protection afforded by the antiserum was possibly caused by induction of phagocytosis in vivo. Bacteria resistant to the lytic activity of normal serum and resistant to phagocytosis are often capsulated [5-7], and negative staining with India ink revealed that the strain used in this study was capsulate, corroborating the above results (picture not shown).

These findings demonstrate that the pre-treatment with Con-A protected mice against experimental infection 
by $S$. marcescens, and support the hypothesis that activation of phagocytes constituted the major, if not the only, early mechanism of defence against $S$. marcescens.

\section{References}

1. Jessop HL, Lambert PA. The role of surface polysaccharide in determining the resistance of Serratia marcescens to serum killing. J Gen Microbiol 1986; 132: 2505-2514.

2. Joiner KA, Schmetz MA, Goldman RC, Leive L, Frank MM. Mechanism of bacterial resistance to complement-mediated killing: inserted C5b-9 correlates with killing for Escherichia coli $0111 \mathrm{~B} 4$ varying in $\mathrm{O}$-antigen capsule and $\mathrm{O}$-polysaccharide coverage of lipid A core oligosaccharide. Infect Immun 1984; 45: $113-117$.

3. Taylor PW. Bacterial and bacteriolytic activity of serum against gram-negative bacteria. Microbiol Rev 1983; 47: 46-83.

4. Muschel LH, Larsen LJ. The sensitivity of smooth and rough gram-negative bacteria to the immune bactericidal reaction. Proc Soc Exp Biol Med 1970; 133: 345-348.

5. Domenico P, Salo RJ, Cross AS, Cunha BA. Polysaccharide capsule-mediated resistance to opsonophagocytosis in Klebsiella pneumoniae. Infect Immun 1994; 62: 4495-4499.

6. Allen PM, Fisher D, Saunders JR, Hart CA. The role of capsular polysaccharide $\mathrm{K} 2 \mathrm{lb}$ of Klebsiella and of the structurally related colanic-acid polysaccharide of Escherichia coli in resistance to phagocytosis and serum killing. $J \mathrm{Med}$ Microbiol 1987; 24: 363-370.

7. Lee C-J. Bacterial capsular polysaccharides - biochemistry, immunity and vaccine. Mol Immunol 1987; 24: 1005-1019.

8. Jessop HL, Lambert PA. Immunochemical characterization of the outer membrane complex of Serratia marcescens and identification of the antigens accessible to antibodies on the cell surface. J Gen Microbiol 1985; 131: 2343-2348.

9. Vuopio-Varkila J, Mäkelä PH. Killing of Escherichia coli in the peritoneal cavity of convalescent mice; role of specific and non-specific immune mechanisms. J Med Microbiol 1988; 25: $205-211$.

10. Kumagai Y, Okada K, Ishimaru T, Sawae Y, Kuroiwa A,
Nomoto K. Effects of vaccination against systemic Serratia infection. J Clin Lab Immunol 1989; 29: 125-132.

11. Kumagai Y, Okada K, Sawae Y. The effect of humoral and cell-mediated immunity in resistance to systemic Serratia infection. J Med Microbiol 1992; 36: 245-249.

12. Felipe I, Bim S, Somensi CC. Increased clearance of Candida albicans from the peritoneal cavity of mice pretreated with concanavalin A or jacalin. Braz J Med Biol Res 1995; 28: $477-483$

13. Vaudaux $P$, Waldvogel FA. Gentamicin antibacterial activity in the presence of human polymorphonuclear leukocytes. Antimicrob Agents Chemother 1979; 16: 743-749.

14. Tabrizi SN, Robins-Browne RM. Elimination of extracellular bacteria by antibiotics in quantitative assays of bacterial ingestion and killing by phagocytes. $J$ Immunol Methods 1993; 158: $201-206$.

15. Felipe 1, Bim S, Loyola W. Participation of mannose receptor on the surface of stimulated macrophages in the phagocytosis of glutaraldehyde-fixed Candida albicans, in vitro. Braz J Med Biol Res 1989; 22: 1251-1254.

16. Pacheco-Soares C, Gaziri LCJ, Loyola W, Felipe I. Phagocytosis of enteropathogenic Escherichia coli and Candida albicans by lectin-like receptors. Braz J Med Biol Res 1993; 25: $1015-1024$.

17. Edwards PR, Ewing WH. Identification of Enterobacteriaceae, 3rd edn. Minneapolis, Burgess, 1972; 311-316.

18. Pelkonen S, Fine JA. Rapid turbidimetric assay for the study of serum sensitivity of Escherichia coli. FEMS Microbiol Lett 1987; 42: $53-57$.

19. Ferrante A, Staugas RE, Rowan-Kelly B et al. Production of tumor necrosis factor alpha and beta by human mononuclear leukocytes stimulated with mitogens, bacteria, and malarial parasites. Infect Immun 1990; 58: 3996-4003.

20. Moscardi-Bacchi M, Brummer E, Stevens DA. Support of Paracoccidioides brasiliensis multiplication by human monocytes or macrophages: inhibition by activated phagocytes. $J$ Med Microbiol 1994; 40: 159-164.

21. Furriel RPM, Lucisano YM, Mantovani B. Precipitated immune complexes of $\operatorname{IgM}$ as well as of $\operatorname{IgG}$ can bind to rabbit polymorphonuclear leucocytes but only the immune complexes of IgG are readily phagocytosed. Immunology 1992; 75: $528-534$. 